\title{
Case Reports in Orthopedic Research
}

\section{Suture Bridge Repair of Proximal Hamstring Avulsion: A Case Report}

\author{
Chad A. Edwards ${ }^{\mathrm{a}}$ Brian H. Goldman ${ }^{\mathrm{a}}$ Andrew Hadeed \\ Daniel Kalbac ${ }^{\mathrm{b}}$ \\ aDepartment of Orthopedic Surgery, Larkin Community Hospital, South Miami, FL, USA; \\ bOrthopedic Surgery, Orthopaedic and Sports Medicine Center of Miami, South Miami, FL, USA
}

\section{Keywords}

Avulsion $\cdot$ Hamstring $\cdot$ Sports medicine $\cdot$ Suture anchor $\cdot$ Tendon repair

\begin{abstract}
Proximal hamstring avulsion injuries can represent a challenging problem and lead to severe patient morbidity if not treated appropriately. The most common method for acute surgical fixation involves the use of between two and five suture anchors in a variety of configurations. The five-anchor fixation has become the gold standard due to the increased biomechanical stability. However, the technique has also been criticized due to the need for greater exposure and technical difficulty. We report on an individual with a proximal hamstring avulsion injury who was managed with a modified approach, utilizing only three anchors in a suture bridge technique. The patient had good clinical outcomes at their 3-month follow-up visit, demonstrating this technique to be a viable option that is less technically demanding.
\end{abstract}

(C) 2020 The Author(s)

Published by S. Karger AG, Basel

\section{Introduction}

Proximal hamstring injuries can represent a challenging problem and lead to severe patient morbidity if not treated appropriately $[1,2]$. These injuries typically occur with rapid acceleration and deceleration with eccentric loads to the hamstring muscle [1,2]. Myotendinous strains are more common than avulsions and typically occur 12 centimeters distal to the ischium, at the myotendinous junction $[2,3]$. These can often be treated conservatively with 
protected weight bearing [4]. True proximal hamstring avulsions only account for three to eleven percent of all hamstring injuries. However, failure to address these injuries can lead to chronic pain, weakness, poor endurance, and sciatica due to tethering of the sciatic nerve [1].

The most common method for acute surgical fixation involves the use of between two and five suture anchors in a variety of configurations [5]. In a biomechanical cadaver study, Hamming et al. [6] compared the use of a variety of sizes and quantity of anchors. It was determined that the use of five small anchors provided more than twice the load to failure compared to the other subgroups. This five-anchor fixation has become the gold standard for fixation due to the increased biomechanical stability. However, the technique has also been criticized due to the need for greater exposure and technical difficulty $[2,7]$.

\section{Case Presentation}

Our patient is a 46-year-old male runner who experienced a "pop" in the hamstring while running. He immediately experienced pain and difficulty with ambulation. On physical exam, he was noted to have extensive bruising along the posterior thigh along with a palpable defect near the proximal hamstring insertion. MRI demonstrated complete avulsion of the proximal hamstring tendon with inferior retraction of $4 \mathrm{~cm}$ (Fig. 1). The patient was scheduled for acute surgical fixation within 2 weeks of the injury.

\section{Technique (Fig. 2)}

With the patient in the prone position, an incision was made horizontally along the gluteal fold. Note that a longitudinal incision can be used for chronic proximal hamstring avulsion injuries to provide greater exposure at the expense of cosmesis. The gluteus maximus muscle was identified via the oblique fibers noted to be in contact with the longitudinal fibers of the hamstring muscles. The inferior aspect of the gluteus muscle was retracted proximally followed by dissection through the deep fascia. The insertion site of the proximal hamstring tendon on the ischial tuberosity was palpated. The sciatic nerve was identified and protected just lateral and deep to the hamstring muscle origin. Fibrous tissue was cleared around the ischial tuberosity and the tuberosity was decorticated to bleeding bone. The retracted hamstring tendon was identified and mobilized utilizing blunt dissection. Two 4.75-mm SwiveLock anchors were placed in the medial and inferolateral aspect of the ischial tuberosity (Fig. 2). With a free needle, a combination of FiberWire and FiberTape were passed through the tendon and tied sequentially using half hitches. A third $4.75-\mathrm{mm}$ SwiveLock anchor was placed proximally in the ischial tuberosity. Utilizing FiberTape, sutures were passed from the first two anchors to the third anchor and secured. Excellent fixation was obtained.

\section{Outcome}

The patient followed the postoperative hamstring rehabilitation program as outlined by Chakravarthy et al. [8]. In phase I after surgery, the patient was placed in a hinged knee brace locked in 90 degrees of flexion and was made non-weight-bearing for 2 weeks. During phase II, the patient began isotonic prone knee flexion from 90 degrees to full flexion with passive extension. During phase II, the patient also began a gradual stretching and strengthening program [8]. At four weeks, the patient was able to range the knee from zero to 90 degrees and began partial weight-bearing at this time. At 8 weeks, the patient was released to return to work and daily activities with a complete return to sports and athletic activities by 12 weeks. The patient progressed quickly and reported feeling no limitations at his 3-month follow-up 
visit. Utilizing the LEFS (Lower Extremity Functional Scale), the patient scored a maximum of 80 points, corresponding to $100 \%$ performance.

\section{Discussion}

While there is a lack of consensus on when operative intervention is warranted, Cohen et al. [2] recommended specific indications. Patients can be treated conservatively if the avulsion involves two or fewer tendons with less than $2 \mathrm{~cm}$ of retraction [2]. Operative intervention should be considered on any patient with greater than $2 \mathrm{~cm}$ retraction of two tendons, or any complete three-tendon avulsions regardless of retraction distance [2]. However, conflicting data do exist. Sallay et al. [9] reported on 12 cases of proximal hamstring avulsions treated nonoperatively with only $58 \%$ of individuals returning to sport, and all at a lower activity level. In a similar study, Malliaropoulos et al. [10] reported on 11 high-level athletes treated nonoperatively with a $100 \%$ return to sport. In a systematic review, Harris et al. [11] described a return to sport rate of $82 \%$ in operative groups versus only $14 \%$ in nonoperative groups.

There is some debate regarding the timing of surgical fixation of proximal hamstring avulsions, although the general consensus is that earlier fixation is superior. Cohen et al. [2] reported on 52 surgically managed proximal hamstring injuries, 40 repaired acutely (within 4 weeks of injury) and 12 repaired after the acute phase. It was concluded that all patients had successful outcomes; however, the patients repaired acutely had higher functional scores and greater strength at follow-up [2]. Supporting this position, Subbu et al. [7] reported on a series of 108 patients stratified into acute (less than weeks), delayed ( 6 weeks through 6 months), and late (after 6 months). They found that while $96.4 \%$ of patients eventually returned to sport regardless of timing to fixation, there was an average of 13 weeks earlier return to sport in the "early" group versus the "late" group [7]. Despite good outcomes with both acute and chronic proximal hamstring fixation, it is generally accepted that chronic (greater than 4 weeks) fixation is more technically difficult, requires more soft tissue releases, requires a larger incision, is more likely to require graft adjunct, and often requires neurolysis due to extensive scar tissue formation around the sciatic nerve [3, 4, 12].

There are multiple techniques for hamstring fixation described in the literature. For instance, Blakeney et al. [13] described a novel technique for fixation utilizing staples. Unfortunately, despite successful outcomes, the technique was not recommended due to high reoperation rates for subsequent staple removal [13]. Cain et al. [5] recently described a technique of unicortical single or double suture button fixation, with successful outcomes in three patients. However, there is some concern regarding the potential need for intraoperative radiographic imaging to ensure proper button positioning in their approach [5]. Similar to our technique presented here, these researcher's approaches require further research into the shortand long-term outcomes.

In this case presentation, we present a modified approach for repairing a proximal hamstring avulsion, utilizing only three anchors in a suture bridge technique. Maximizing the footprint increases stability of the repair without the need for additional suture anchors. In addition to decreasing technical difficulty, using fewer anchors reduces cost when compared to the standard five-anchor technique $[5,14]$. The disadvantage of using three suture anchors is the potential for decreased mechanical strength. Although there is no biomechanical study comparing the two techniques, there is reason to believe that using fewer anchors translates to inferior strength based on Hamming et al.'s study [6]. Biomechanical studies comparing 
three versus five suture anchors would be beneficial in elucidating the optimal configuration for hamstring suture anchor repair. The patient was informed that their case would be submitted for publication, and he provided consent.

\section{Conclusion}

Despite extensive and often contradicting literature on the management of proximal hamstring injuries, it is clear there is a role for surgical intervention. With multiple methods of fixation, we have presented a technique that can decrease cost by utilization of only three anchors. This case has demonstrated an excellent result from this technique with full return to sport at only 3 months follow-up. Additional research is necessary to assess the biomechanical strength of this construct. Furthermore, a larger patient pool will be needed to determine complications and success rates of this new technique.

\section{Statement of Ethics}

Informed consent was obtained authorizing treatment and documentation of clinical results. A thorough discussion was had with the patient regarding publication of their case, to which they consented.

\section{Disclosure Statement}

The authors of this article have no conflicts of interest to declare.

\section{Funding Sources}

No specific funding was received for this study.

\section{Author Contributions}

Authors Chad Edwards, Brian Goldman, Andrew Hadeed, and Daniel Kalbac were involved in the following capacities: substantial contributions to the conception or design of the work; or the acquisition, analysis, or interpretation of data for the work; AND drafting the work or revising it critically for important intellectual content; AND final approval of the version to be published; AND agreement to be accountable for all aspects of the work in ensuring that questions related to the accuracy or integrity of any part of the work are appropriately investigated and resolved.

The following authors were involved in specific aspects of writing the paper: Chad Edwards: writing the technique, outcome, discussion, and conclusion, and made edits, Brian Goldman: writing the abstract, technique, outcome, discussion, and conclusion, and editing, Andrew Hadeed: writing the outcome, discussion, making image artwork for the technique description, Daniel Kalbac: writing the technique, outcome, discussion, and making edits to the paper. 


\section{Case Reports in Orthopedic Research}

\section{References}

1 Carmichael J, Packham I, Trikha SP, Wood DG. Avulsion of the proximal hamstring origin. Surgical technique. J Bone Joint Surg Am. 2009 Oct;91 Suppl 2:249-56.

2 Cohen SB, Rangavajjula A, Vyas D, Bradley JP. Functional results and outcomes after repair of proximal hamstring avulsions. Am J Sports Med. 2012 Sep;40(9):2092-8.

3 Klingele KE, Sallay PI. Surgical repair of complete proximal hamstring tendon rupture. Am J Sports Med. 2002 Sep-Oct;30(5):742-7.

4 van der Made AD, Reurink G, Gouttebarge V, Tol JL, Kerkhoffs GM. Outcome After Surgical Repair of Proximal Hamstring Avulsions: A Systematic Review. Am J Sports Med. 2015 Nov;43(11):2841-51.

5 Cain MT, Jones MC, Reed DN. A Novel Technique to Repair Acute and Chronic Proximal Hamstring Avulsions Orthopedics. 2018 Mar;41(2):116-9.

6 Hamming MG, Philippon MJ, Rasmussen MT, Ferro FP, Turnbull TL, Trindade CA, et al. Structural properties of the intact proximal hamstring origin and evaluation of varying avulsion repair techniques: an in vitro biomechanical analysis. Am J Sports Med. 2015 Mar;43(3):721-8.

7 Subbu R, Benjamin-Laing H, Haddad F. Timing of surgery for complete proximal hamstring avulsion injuries: successful clinical outcomes at 6 weeks, 6 months, and after 6 months of injury. Am J Sports Med. 2015 Feb;43(2):385-91.

8 Chakravarthy J, Ramisetty N, Pimpalnerkar A, Mohtadi N. Surgical repair of complete proximal hamstring tendon ruptures in water skiers and bull riders: a report of four cases and review of the literature. $\mathrm{Br}$ J Sports Med. 2005 Aug;39(8):569-72.

9 Sallay PI, Friedman RL, Coogan PG, Garrett WE. Hamstring muscle injuries among water skiers. Functional outcome and prevention. Am J Sports Med. 1996 Mar-Apr;24(2):130-6.

10 Malliaropoulos N, Psyllakis P, Tsitas K, Papalada A. Conservative Treatment of Total Proximal, Non-Avulsion, Hamstring Muscle-Rupture, in High Level Athletes. Br J Sports Med. 2013 Oct;47(10):e3.4-e3.

11 Harris JD, Griesser MJ, Best TM, Ellis TJ. Treatment of proximal hamstring ruptures - a systematic review. Int J Sports Med. 2011 Jul;32(7):490-5.

12 Moatshe G, Chahla J, Vap AR, Ferrari M, Sanchez G, Mitchell JJ, et al. Repair of Proximal Hamstring Tears: A Surgical Technique. Arthrosc Tech. 2017 Mar;6(2):e311-7.

13 Blakeney W, Zilko S, Chiri W, Annear P. A Novel Technique for Proximal Hamstring Tendon Repair: High Reoperation Rate in a Series of 56 Patients. Advances in Orthopedic Surgery. 2014;2014:1-6.

14 Meier SW, Meier JD, Levy AS. Rotator Cuff Repair: The Effect of Double-Row Fixation versus Single-Row Fixation on Three-Dimensional Repair Site (SS-03). Arthroscopy. 2004;20:e2.
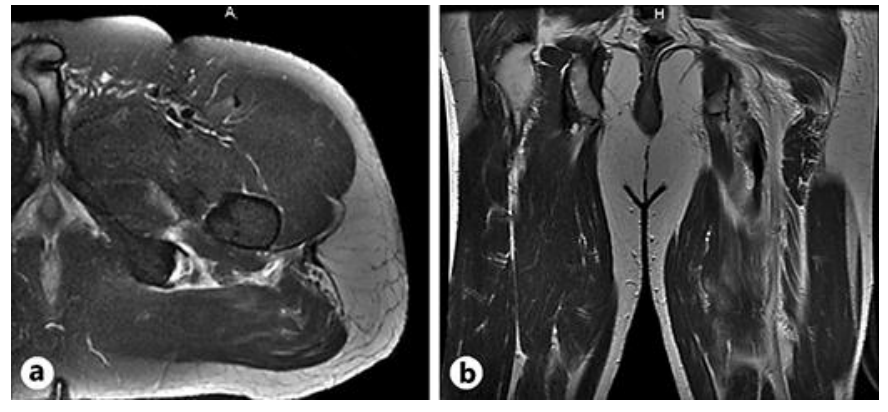

Fig. 1. Axial (a) and coronal (b) MRI cuts demonstrating complete tear of the left hamstring tendon avulsed from the ischial tuberosity. The distal tendon edge is retracted inferiorly by approximately $4 \mathrm{~cm}$. 

Research

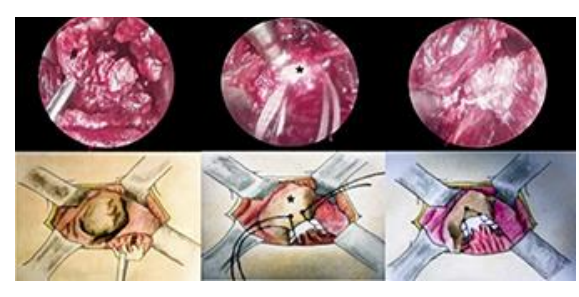

Fig. 2. Intraoperative avulsion of the proximal hamstring with suture anchor configuration and final fixation of the hamstring tendon (\#) to the ischial tuberosity $(*)$. 